\title{
Kurzbeiträge
}

\author{
Thomas Elbel
}

\section{Gibt es eine Pflicht zur Bedarfsdeckung bei einer zentralisierten Beschaffungsbehörde?}

Beschaffungsbehörde; Bedarfsträger; Skaleneffekt; In-House-Geschäft; Wirtschaftlichkeit; Unzulässige Markterkundung

Der Artikel untersucht die Frage, inwieweit die Parallelausschreibung des bezugsberechtigten Bedarfsträgers einer zentralisierten Beschaffungsbehörde notwendigerweise zu unwirtschaftlichen Ergebnissen führt und damit eventuell einen Rechtsverstoß darstellt, aus dem sich im Umkehrschluss eine Pflicht zur Unterlassung konstruieren lässt.

\section{Einleitung}

\section{Abstrakte Themenstellung}

In diesem Artikel möchte ich untersuchen, inwieweit dem Recht zur vergaberechtsfreien Bedarfsdeckung bei einer zentralisierten Beschaffungsbehörde auch die Pflicht folgt, von diesem Recht Gebrauch zu machen.

Zentralisierte Beschaffungsbehörden decken die Bedarfe ihres aus anderen Behörden bestehenden Kundenkreises durch Sammelausschreibungen. Der Sinn eines solchen Konstrukts liegt im Wesentlichen in der Erzielung von Ersparnissen, die durch die Zusammenfassung der Bedarfe mehrerer Kundenbehörden entstehen. Bestünde keine Pflicht der Kundenbehörden, ihre Bedarfe aus den durch solche Sammelausschreibungen erzielten Verträgen zu decken, so könnte dies drei Probleme aufwerfen. Erstens könnte ein vergaberechtliches Problem entstehen, indem sich aus Sicht der Bieter der Sammelausschreibung der durch diese Ausschreibung repräsentierte tatsächliche Bedarf nicht abschätzen lässt und ihnen damit im Rahmen der Preiskalkulation ein unzumutbares Wagnis auferlegt wird. Ein Folgeproblem könnte dann dadurch entstehen, dass diesem Wagnis seitens der Bieter durch eine eigentlich unnötige Preiserhöhung begegnet wird. Schließlich könnte dadurch drittens der durch die Sammelausschreibung beabsichtigte Effekt einer gegenüber Einzelausschreibungen wirtschaftlicheren Beschaffung wesentlich verringert oder gar zunichte gemacht werden.

Es ist darüber hinaus anzunehmen, dass die Gesamtproblematik einen selbstverstärkenden Charakter hat. Wenn nämlich die Sammelausschreibung gegenüber einer Einzelausschreibung erwartete Preisvorteile nicht oder nicht in dem erwarteten Maße zur Folge hat, steigt damit wiederum 
die Tendenz der Kundenbehörden zum Ausstieg aus dem System der zentralisierten Beschaffung. Am Ende droht damit das System der zentralisierten Beschaffungsbehörde selbst ad absurdum geführt zu werden.

Es könnte also sein, dass eine Pflicht der Kundenbehörden zur Bedarfsdeckung aus Sammelausschreibungen einer zentralisierten Beschaffungsbehörde alternativlos ist, um Skaleneffekte möglichst effizient realisieren zu können. Fraglich ist allerdings, ob die effiziente Realisierung der Skaleneffekte auch rechtlich alternativlos ist, z. B. weil sich eine Pflicht zu ihrer Realisierung aus dem haushaltsrechtlichen Gebot der Wirtschaftlichkeit und Sparsamkeit herleiten lässt. Dies zu untersuchen, ist das Hauptanliegen des vorliegenden Artikels.

\section{Fallbeispiel}

Zunächst möchte ich die Fragestellung anhand eines realen Fallbeispiels verdeutlichen. Das ITDienstleistungszentrum (ITDZ) ist eine rechtsfähige Anstalt öffentlichen Rechts des Landes Berlin. Das ITDZ hat gem. § 2 Abs. 2 ITDZG (v. 19. November 2004, GVB1 Bln, S. 460.) unter anderem die Aufgabe, ,den Stellen des Landes Berlin ein umfassendes Angebot an Informationstechnik, -anwendungen und -dienstleistungen zur Verfügung“ zu stellen. Gemäß Abs. 3 sind die Stellen des Landes Berlin berechtigt, „unmittelbar“, d. h. „ohne dass es eines besonderen Vergabeverfahrens bedarf" Leistungen der Anstalt zu beziehen. Das ITDZ selbst wiederum beschafft gem. Abs. 5 Leistungen ,nach Maßgabe der für öffentliche Auftraggeber geltenden Vergabevorschriften“. Kurz gesagt bündelt das ITDZ also die IT-Bedarfe der Berliner Behörden, schreibt die gebündelten Bedarfe aus und verkauft diese dann an die Bezugsberechtigten. Allerdings sind die Behörden des Landes Berlin lediglich in Ausnahmefällen zur Deckung ihrer einschlägigen Bedarfe beim ITDZ verpflichtet; und zwar nur dann, wenn das Land Berlin zuvor eine entsprechende Landesvereinbarung mit dem ITDZ geschlossen und eine Abnahmeverpflichtung seiner Behörden durch entsprechende Weisung festgelegt hat (vgl. insoweit lit. E der Einleitung zum Gesetzesentwurf des ITDZG in Drs. des Abgeordnetenhauses von Berlin Nr. 15/3080 vom 18. August 2004, S. 2 sowie Ziffer 5 Abs. 1 u. 3 der Verwaltungsvorschriften für die Steuerung des IT-Einsatzes in der Berliner Verwaltung [VV IT-Steuerung] vom 17. August 2004).

In der Praxis ist es daher so, dass manche der bezugsberechtigten Behörden ihre Bedarfe selbstständig ausschreiben. Dies gilt mitunter sogar dann, wenn sie diese Bedarfe zuvor beim ITDZ zur gebündelten Ausschreibung angemeldet haben. Motiv ist häufig die Überzeugung, im Alleingang wirtschaftlichere Ergebnisse erzielen zu können.

\section{Aufbau der Untersuchung}

In der nachfolgenden Untersuchung dieser Problematik werde ich zunächst das Konstrukt einer zentralisierten Beschaffungsbehörde abstrakt beschreiben (nachfolgend unter II. 1) und dabei einige reale Beispiele solcher Beschaffungsbehörden benennen. Daran anschließend will ich die vergaberechtlichen Regeln aufzeigen, die die Einrichtung solcher Behörden begünstigt und die Reichweite dieser Regeln aufzeigen (nachfolgend unter II. 2.). Im folgenden Teil werde ich kurz 
auf die wirtschaftlichen Mechanismen namentlich die Skaleneffekte eingehen, die die Einrichtung solcher Behörden in vielen Fällen sinnvoll erscheinen lassen (nachfolgend unter II. 3). Im Kernteil der Arbeit werde ich dann untersuchen, inwieweit sich aus dem Recht, d. h. insbesondere dem in den Haushalts- und Vergabegesetzen normierten Wirtschaftlichkeitsgrundsatz eine Pflicht zur Bedarfsdeckung bei zentralisierten Beschaffungsbehörden entnehmen lässt bzw. ob andere gesetzliche Vorschriften dem entgegenstehen (nachfolgend unter III.).

\section{Rechtliche und ökonomische Problemgrundlagen}

\section{Zentralisierte Beschaffungsbehörde}

Es gibt eine seit Jahrzehnten zu beobachtende Tendenz größerer Behördenstrukturen, ihre Bedarfe gebündelt auszuschreiben. Hierzu werden häufig zentralisierte Strukturen, d. h. Beschaffungsämter gebildet (z. B. das jüngst errichtete Bundesamt für Ausrüstung, Informationstechnik und Nutzung der Bundeswehr). Häufig erhalten diese Behörden einen eigenen Haushalt, z. B. durch Überführung in einen Eigenbetrieb oder Landesbetrieb nach der jeweils einschlägigen LHO (z. B. der Landesbetrieb Information und Technik Nordrhein-Westfalen). Mitunter werden die Beschaffungsbehörden auch rechtlich verselbstständigt, indem sie entweder privatisiert werden (z. B. die Kommunale Informationsdienste Magdeburg $\mathrm{GmbH}$ ), oder als juristische Person des öffentlichen Rechts, also zumeist in der Form einer Anstalt ausgegliedert werden (Elbel 2008, S. 432 f.), wie das ITDZ Berlin. Während Streitigkeiten zwischen den Beschaffungseinheiten und ihren Bedarfsträgern bei rein haushaltsmäßiger Verselbstständigung Insichprozesse sind, verleiht ihnen die rechtliche Verselbstständigung die Fähigkeit gegen diese im eigenen Namen zu prozessieren, selbst wenn beide Behörden unter der Rechts- und Fachaufsicht einer gemeinsamen Behördenspitze stehen (Elbel 2008, S. 437). Die zwischen einer rechtlich selbstständigen und öffentlich-rechtlich verfassten Beschaffungsbehörde und ihren ebenfalls öffentlichen Bedarfsträgern geschlossenen Lieferungs- und Leistungsverträge sind in aller Regel zivilrechtlicher Natur (Elbel 2010, S. 13ff.). Bei rechtlicher Unselbstständigkeit sind die in derartigen Vereinbarungen geregelten Pflichten hingegen rein politischer Natur und rechtlich nicht einklagbar (Elbel 2008, S. 437).

Am deutlichsten beobachtbar ist die vorbeschriebene Tendenz zur Beschaffungsbündelung auf der kommunalen und Landesebene und dort insbesondere im Bereich der Informationstechnik (so zählt z. B. die Bundes-Arbeitsgemeinschaft der Kommunalen IT-Dienstleister ViTaKo e. V. hat zurzeit dieser Veröffentlichung gut fünfzig Mitglieder bei steigender Tendenz). Ähnliche Strukturen gibt es allerdings auch im Bereich der Bundesverwaltung. Das Kaufhaus des Bundes ist laut eigener Darstellung eine Plattform über welche ,die Behörden und Einrichtungen des Bundes elektronische Bestellungen aus Rahmenvereinbarungen tätigen können, welche die Zentrale Beschaffungsstellen abgeschlossen haben“. Hinter dem Begriff der Zentralen Beschaffungsstellen verbergen sich das Bundesamt für Ausrüstung, Informationstechnik und Nutzung der Bundeswehr, die Bundesfinanzdirektion Südwest, die Bundesanstalt für Materialforschung und -prüfung 
und insbesondere das Beschaffungsamt des Bundesministeriums des Innern, das diese Plattform betreibt.

Quantitative wirtschaftswissenschaftliche Untersuchungen des staatlichen zentralisierten Beschaffungswesens, insbesondere der Marktanteile in bestimmten Märkten und das Verhältnis dieser Marktanteile zu jenen nichtzentralisierter Beschaffungen sind zurzeit der Abfassung dieses Artikels noch nicht vorhanden (eine ältere, rein qualitative Übersicht zur Tendenz der Berliner Behörden zu mit Sammelausschreibungen des ITDZ konkurrierenden Einzelausschreibungen findet sich in Anlage 2, Teil 1 zur Kleinen Anfrage des Abg. Kaczmarek, Drs. 15/11601 des Abgeordnetenhauses von Berlin).

\section{Vergaberechtliche Situation der Beschaffungsbehörden}

Zwischen jenen Beschaffungsbehörden, die im Verhältnis zu ihren Bedarfsträgern rechtlich selbstständig sind, gilt im Verhältnis zu diesen grundsätzlich Vergaberecht, denn es ist für die Erfüllung der Definition des öffentlichen Auftrages i.S.d. § 99 Abs. 1 GWB grundsätzlich unmaßgeblich, ob der Beauftragte selbst dem hoheitlichen Bereich zuzurechnen ist. Hiervon gilt allerdings eine wesentliche Ausnahme: Das sogenannte In-House-Privileg, das der EuGH erstmalig in der Teckal-Entscheidung formuliert hat (EuGH v. 18. November 1999, Slg. 1999, I-8156). Demzufolge unterfällt die Beauftragung einer Behörde dann nicht unter das Vergaberecht, wenn diese vom Auftraggeber ,wie eine eigene Dienststelle“ kontrolliert wird und sie ihre Tätigkeit im Wesentlichen für eben diesen Auftraggeber verrichtet (EuGH a. a. O., Rd. 50).

Während die Urentscheidung also nur die direkte, rein vertikale Beziehung zwischen Auftraggeber und Auftragnehmer privilegiert, lässt sich der dieser Entscheidung und ihren Folgeentscheidungen zugrundeliegenden Philosophie entnehmen, dass eine In-House-Situation auch dann vorliegen kann, wenn beide Stellen (direkt oder indirekt) von ein und derselben „Mutter-Behörde“ beherrscht werden, ja sogar dann, wenn die „Tochter“ die „Mutter“ beauftragt (Elbel 2011, S. 185ff.). Dies folgt in letzter Konsequenz aus der insbesondere von Hardraht in seiner Dissertation vertretenen Theorie der ,funktionellen Identität“ als Voraussetzung der In-House-Situation, die auch diesem Artikel zugrunde gelegt werden soll. Diese Theorie unterteilt von „Teckal“ ausgehend die Beschaffungen der öffentlichen Hand in einen Binnen- und einen Außenbereich. Vergaberecht soll demzufolge im Binnenbereich auch dann nicht anwendbar sein, wenn zwischen Auftraggeber und -nehmer eine funktionelle Identität bestehe, denn in diesem Fall sei der Auftragnehmer begrifflich noch als Teil des öffentlichen Auftraggebers im Sinne des § 99 I GWB selbst anzusehen (Hardraht 2006, S. 198ff. u. S. 299 f.).

Es muss allerdings an dieser Stelle angemerkt werden, dass diese Auslegung der Teckal-Grundsätze keinesfalls unumstritten ist. Insofern ist die häufig gelebte Praxis zentralisierter Beschaffungsbehörden, Vergaben in Form sogenannter Rahmenausschreibungen vorzunehmen, aus denen sich dann andere rechtlich selbstständige Behörden eines größeren Verbundes vergabefrei bedienen können (vergleiche insofern z. B. die Praxis des eingangs erwähnten Kaufhauses des Bundes oder der länder- und kommunenübergreifenden Anstalt Dataport) vergaberechtlich zumindest zweifelhaft. Das gilt, wegen des Vorrangs des sekundären Europarechts natürlich auch für Gesetze, die eine solche Praxis festzuschreiben trachten, namentlich den eingangs zitierten 
$\S 2$ Abs. 3 S. 1 ITDZ-G, der allen Stellen des Landes Berlin eine vergabeverfahrensfreie Beauftragung des ITDZ erlaubt (vergleichbare Regelungen finden sich indes auch in $\S 3$ Abs. $1 \mathrm{~S} .1$ DVZG M-V sowie $\S 3$ Abs. 7 des Gesetzes zu dem Staatsvertrag vom 30. September 2008 zwischen dem Land Berlin und dem Land Brandenburg über die Errichtung eines Landeslabors Berlin-Brandenburg).

Allerdings deuten jüngere Entscheidungen des EuGH eher darauf hin, dass Hardrahts Theorie durchaus mit der generellen Rechtsprechungspraxis des Gerichts übereinstimmt (Elbel 2011, S. 192 f.). So hat z.B. der EuGH in seiner „Carbotermo“-Entscheidung (EuGH v. 11. Mai 2006, Slg. 2006, I-4137) festgestellt, dass eine In-House-Situation auch im Falle einer indirekten Beherrschung der Auftragnehmerbehörde durch die Auftraggeberbehörde gegeben sein kann. Die „Teckal“-Entscheidung selbst, wie auch die neueren Entscheidungen „Coditel Brabant“ (EuGH v. 13. November 2008, Slg. 2008, I-8457, abgedr. in EuZW 2009, S. 55) und „Rugenberger Damm“ (EuGH v. 9. Juni 2009, Slg. 2009, I-0000, abgedr. in EuZW 2009, S. 529) nehmen das ebenso für eine Situation an, in der die Auftragnehmerbehörde von mehreren auftragsberechtigten Behörden gemeinsam beherrscht wird. Für Hardrahts Theorie spricht zuguterletzt insbesondere die Entscheidung „Stadt Halle“ (EuGH v. 11. Januar 2005, Slg. 2005 I-1, abgedr. in EuZW 2005, S. 86), in der der EuGH für den Verbleib einer Beschaffungsentscheidung im vergabefreien Verwaltungsbinnenraum insbesondere die Interesseneinheit der Parteien anführt.

\section{Wirtschaftlicher Sinn der Bündelung von Einkaufskapazität}

Warum ist es überhaupt vorteilhaft, die Beschaffungsbedarfe mehrerer Behörden zu bündeln? Die Antwort liegt im wirtschaftswissenschaftlichen Prinzip der „Economies of Scale“, auf Deutsch auch als Skaleneffekte bezeichnet. Gemeint ist hiermit zunächst einmal der Ersparniseffekt, den Unternehmen durch die Produktion höherer Stückzahlen erzielen. Da die durch die Produktion verursachten Fixkosten (z. B. Kosten des Produktdesigns) im Gegensatz zu den variablen Kosten gleich bleiben, reduzieren sich mit steigendem Ausstoß die als Stückkosten bezeichneten Produktionskosten pro Stück (Wildmann 2010, S. 214 f.). Bestellen Kunden Produktmengen, die diesen Effekt besonders signifikant ausfallen lassen, so wächst die unternehmerische Neigung, die interne Ersparnis durch entsprechende Rabattierung zumindest zum Teil an den Kunden weiterzureichen. Die Bündelung der Bedarfe mehrerer Behörden wird sich also häufig in niedrigeren Stückpreisen niederschlagen. Die zunehmende Industrialisierung des Marktes für Informationstechnik in den Bereichen Hardware, Software und Services hat eine Zunahme von Skaleneffekten in diesem Markt zur Folge (Walter/Böhmann/Krcmar 2007, S. 6ff.).

Ein weiterer positiver Effekt ist die Tatsache, dass - angesichts der vorbeschriebenen vergaberechtlichen Konstellation - bei Bündelung mehrerer Bedarfe durch eine zentrale Beschaffungsbehörde der mitunter nicht unbeträchtliche (hauptsächlich personelle) Aufwand einer Ausschreibung nur einmal entsteht. Auch dies sind gewissermaßen Fixkosten, die die Stückkosten aus Sicht des Beschaffers senken.

Weitere Ersparniseffekte entstehen dann langfristig dadurch, dass die Bedarfsträger die für die Beschaffung notwendige Expertise personell nicht mehr in einem Umfang vorhalten müssen, der für eine eigene Ausschreibung notwendig ist. 
Diese Ersparnisse werden mitunter dadurch ein wenig gemindert, dass die zentralisierte Beschaffungsbehörde einen eigenen Deckungsbeitrag auf den bei der Beschaffung erzielten Stückpreis aufschlägt, (vgl. insoweit die Antwort auf die Kleine Anfrage des Abg. Kaczmarek, Drs. 15/11601 des Abgeordnetenhauses von Berlin, Ziffer 4) der idealerweise so berechnet ist, dass er die durch die Bündelung erzielten Ersparnisse aus Sicht des jeweiligen Bedarfsträgers nicht aufzehrt und andererseits den auf der Seite der Beschaffungsstelle entstandenen Aufwand decken kann. Üblicherweise wird dieser Deckungsbeitrag in Prozent auf den prognostizierten Wert der Ausschreibung berechnet und liegt regelmäßig im niedrigen einstelligen Bereich. In der Realität ist gerade die Berechnung dieses Deckungsbeitrages häufig ein Konfliktpunkt (so z. B. in der Entscheidung des Kammergerichts v. 25. Juli 2000, Az.: KartVerg 11/00, Ziffer II. 3.; wobei der Konflikt hier von einer Bieterin ausging) und damit neben der Unzufriedenheit mit der Ersparnisquote (vgl. hierzu z. B. die Kleine Anfrage des Abg. Schruoffenegger v. 30. Januar 2007, Drs. 16/10 303 des Abgeordnetenhauses von Berlin) ein weiteres Motiv, eine eigene Parallelausschreibung durchzuführen.

\section{Zulässigkeit einer Parallelausschreibung}

\section{Haushaltsrechtliche Problematik}

Der Wirtschaftlichkeitsgrundsatz findet sich schon auf verfassungsrechtlicher Ebene in Art. 114 Abs. 2 S. 1 GG und auf der einfachgesetzlichen in $\S 7$ Abs. 1 S. 1 BHO sowie den Parallelvorschriften der LHOen der Länder. Haushaltsrechtlich bedeutet dies die Verpflichtung staatlicher Stellen ,auf ein möglichst günstiges Verhältnis zwischen Ressourceneinsatz und Nutzen.“ (Gröpl 2007, Rn. 11).

Unterstellt, die Bündelung durch eine zentralisierte Beschaffungsbehörde würde durch Skaleneffekte bewirkte Preisreduzierungen ausnahmslos zur Folge haben, müsste die „ungebündelte“ Parallelausschreibung einer bezugsberechtigten Behörde automatisch zu einem weniger wirtschaftlichen Ergebnis führen. Da außerdem die Durchführung der Parallelausschreibung selbst zusätzliche Kosten verursacht, die vor diesem Hintergrund nicht notwendig sind, lässt sich postulieren und prognostizieren, dass eine solche Parallelausschreibung aus Sicht der ausschreibenden Stelle per se unwirtschaftlich ist. Sie ist im Übrigen auch aus der Gesamtsicht des bezugsberechtigten Verbundes unwirtschaftlich, da sie geeignet ist, den durch die Bündelung beabsichtigten Skaleneffekt zu reduzieren, indem sie die gebündelte Stückzahl verringert bzw. den Bietern wegen der Unkalkulierbarkeit der tatsächlichen Abnahmemenge ein größeres Wagnis auferlegt, was sich wieder in Preisaufschlägen niederschlagen dürfte.

Parallelausschreibende Behörden verweisen allerdings - wie bereits eingangs bemerkt - in der Praxis darauf, dass sie oft ähnliche und mitunter sogar bessere Ergebnisse erzielen, als mit der gebündelten Ausschreibung erreicht wurden (eine gute, wenn auch etwas ältere Übersicht über das diesbezügliche Verhalten, und insbesondere die Motivlage innerhalb der Berliner Verwaltung, bietet die bereits erwähnte Anlage 2, Teile 1 und 2 zur Kleinen Anfrage des Abg. Kaczmarek, Drs. 15/11601 des Abgeordnetenhauses von Berlin). Kann man also hieraus folgern, dass die 
Theorie der Skaleneffekte nicht geeignet ist, Prognosen über die Wirtschaftlichkeit einer gebündelten Ausschreibung zu treffen? Ich würde diese Frage eher verneinen und hierzu die Vermutung anstellen, dass der beschriebene Sondereffekt eher dadurch zustande kommt, dass die Verkäufer für die Zwecke der Parallelausschreibung Spekulationspreise bieten, um auf diese Weise die Durchbrechung des aus ihrer Sicht lästigen Monopsons zu fördern. Dieses Verhalten wird dadurch erleichtert, dass die konkurrierende Einzelausschreibung durchführende Behörde meist die Ergebnisse der vorangegangenen Sammelausschreibung in ihre eigenen Einzelausschreibungen einfließen lässt (vgl. insoweit die Antworten der Berliner Behörden auf Frage 8 in Anlage 2, Teil 1 zu der Kleinen Anfrage des Abg. Kaczmarek, Drs. 15/11601 des Abgeordnetenhauses von Berlin). Hierdurch können die Bieter der zeitlich nachlaufenden Einzelausschreibungen ihre Kalkulationen entsprechend anpassen. Spekulationspreise wären zwar aus vergaberechtlichen Gründen auszuschließen (vgl. z. B. § 16 Abs. 6 VOL/A), sind aber in der Realität aus Sicht der ausschreibenden Stelle selten aufdeckbar (Leinemann 2008, S. 346ff.).

Dabei reicht es oft schon aus, dass der Bieter in der Parallelausschreibung einfach denselben Preis bietet, den er auch in der gebündelten bereits geboten hat, da zentralisierte Beschaffungsbehörden zumeist gesetzlich gehalten sind, ihre eigenen Aufwände in Form eines geringen prozentualen Aufschlages auf die erzielten Ausschreibungsergebnisse an ihre Kunden weiterzureichen. Insofern sind Parallelausschreibungen in mehrfacher Weise geeignet, den positiven Effekt der Bündelung von Beschaffungen zu unterlaufen. Es handelt sich dabei jedenfalls im haushaltsrechtlichen Sinn um unwirtschaftliches Verhalten. Das Haushaltsrecht bietet allerdings keinerlei Instrumente, Parallelausschreibungen zu verhindern. Es kann hier lediglich eine Ahndung in Form einer Rüge des zuständigen Rechnungshofs oder durch die zuständige Fachaufsicht erfolgen.

\section{Vergaberechtliche Problematik}

Über $\S 97$ Abs. 5 GWB und entsprechende Regelungen der Verdingungsordnungen beansprucht der Wirtschaftlichkeitsgrundsatz auch im Vergabeverfahren explizite Wirksamkeit. Hieraus folgt in der Regel die Pflicht zur Bezuschlagung des Angebots mit dem besten Preis-Leistungs-Verhältnis (Kirchner 2010, S. 734). Dies gilt allerdings nur soweit nicht eine rein auf den Preis bezogene Bezuschlagung möglich erscheint, was aber bei komplexeren Produkten selten der Fall ist.

In einer Situation, in der den bezugsberechtigten Bedarfsträgern die Wahl zwischen einem Bezug aus der gebündelten Ausschreibung und einer eigenen Parallelausschreibung völlig freigestellt ist, ist primär die gebündelte Ausschreibung angreifbar, da sie eine unzulässige Markterkundung i. S. v. $§ 2$ Abs. 3 VOL/A bzw. $§ 2$ Abs. 4 VOB/A darstellt (KG Berlin v. 15. April 2004, Az.: 2 Verg 22/03, II. 3. a). Denn eine unzulässige Markterkundung im Sinne dieser Vorschriften liegt z. B. auch dann vor, wenn Ausschreibungen dazu genutzt werden, um Preisvergleiche zu anderen Ausschreibungen mit parallelem Ausschreibungsgegenstand zu ziehen (Mestwerdt 2011, S. 42 f.). Wenn man aber im Sinne der o. a. Hardrahtschen Theorie der funktionellen Identität Auftraggeber- und -nehmerbehörde als gemeinsamen staatlichen Binnenbereich betrachtet, so stellt es sich aus der Außensicht so dar, als ob dieselbe Leistung zweimal ausgeschrieben wird, so dass insoweit eine solche unzulässige Markterkundung vorliegt. Dies gilt natürlich umso mehr, 
wenn die die Sammelausschreibung durchführende Stelle gegenüber der parallelausschreibenden Stelle rechtlich unselbstständig ist, wie z. B. im Falle eines Landesbetriebes nach § 26 LHO.

Es ergibt sich im Anwendungsbereich der VOL/A darüber hinaus die paradoxe und volkswirtschaftlich absurde Situation, dass sich - falls die Parallelausschreibung zeitlich nachlaufend stattfindet - die zentralisierte Beschaffungsbehörde als Bieterin an dieser mit guten Erfolgsaussichten beteiligen könnte. Im Bereich der Bauleistungen wäre diese Möglichkeit allerdings wegen $\S 6$ Abs. 1 Ziffer 3 VOB/A nicht gegeben, da diese Vorschrift - anders als die VOL/A - Behörden von der Teilnahme am Wettbewerb um öffentliche Aufträge i. S. d. § 99 Abs. 1 GWB ausschließt.

\section{Ergebnis}

Im Ergebnis ist folgendes festzuhalten:

1. Die vor allem im Bereich der staatlichen Beschaffung von IT-Leistungen übliche Bündelung des Beschaffungsbedarfs mehrerer Bedarfsträger durch eine rechtlich selbstständige, zentralisierte Beschaffungsbehörde ist bei entsprechender Auslegung des vergaberechtlichen InHouse-Privilegs möglich, wenn sowohl alle Bedarfsträger, als auch die Beschaffungsbehörde von einer gemeinsamen „Mutter“ kontrolliert werden und auch das Wesentlichkeitsprinzip erfüllt ist.

2. Eine eigene Parallelausschreibung eines dieser Bedarfsträger wird gemäß des Prinzips der „Economies of Scale“ in aller Regel zu einem unwirtschaftlicheren Ergebnis als die gebündelte Ausschreibung führen. Sie stellt daher einen Verstoß gegen das haushaltsrechtliche Prinzip der Wirtschaftlichkeit und Sparsamkeit dar.

3. Eine solche Parallelausschreibung ist auch vergaberechtlich problematisch. Zum einen provoziert sie die Bieter zu Spekulationspreisen und zum anderen läuft die gebündelte Ausschreibung Gefahr, als unzulässige Markterkundung zu gelten.

4. Hieraus lässt sich eine Pflicht zum Unterlassen solcher Parallelausschreibungen konstruieren, da insoweit regelmäßig eine Ermessensreduzierung auf Null vorliegt. Allerdings dürfte es aus faktischen Gründen unmöglich sein, eine solche Ausschreibung im Vorhinein zu blockieren. Daher wäre es sinnvoll, ein Verbot von parallelen (dezentralen) Ausschreibungen gesetzlich oder mindestens per Verwaltungsvorschrift zu verankern, wie es z. B. in der Beschaffungsordnung der Stadt Hamburg vom 1. Januar 2009 geschehen ist.

Abstract

Thomas Elbel; Are Government Agencies under a Legal Obligation to Cover their Demands via a Centralized Public Procurement Agency?

Public Procurement Agencies; Public Customer Entities; Economies of Scale; In-house-transaction; Economic Efficiency; Illegitimate Market Survey 
Gibt es eine Pflicht zur Bedarfsdeckung bei einer zentralisierten Beschaffungsbehörde?

In a scenario, where a centralized public procurement agency carries out a bidding procedures for its public customer entities, such centralization is a legitimate use of the "in-house-privilege", if all entities concerned are controlled by the same "corporate mother". The centralization of the procurement will produce results which are more economically efficient due to the principle of the economies of scale. If one of the customer entities parallels the centralized bidding procedure with its own effort, this effort is likely to produce less efficient results and is thus a violation of the principle of economic efficiency under budgetary law. It also puts a risk on the centralized bidding procedure, since the latter may be regarded as an illegitimate market survey under public procurement law. Thus in the situation described a duty may be construed to abstain from such a parallel bidding procedure.

\section{Literaturverzeichnis}

Elbel, Thomas (2008), Wann sind Rechtsstreitigkeiten über Leistungsbeziehungen zwischen Beschaffungsbehörden und ihren Bedarfsträgern Insichprozesse? in: DVBl, Heft 7, S. 432-437.

Elbel, Thomas (2010), Rechtsweg bei Lieferungs- und Leistungsverträgen zwischen Beschaffungsbehörden und ihren Bedarfsträgern, in: ZögU, Heft 1, S. 13-30.

Elbel, Thomas (2011), Reichweite der vergaberechtlichen Figur des „In-House-Geschäfts“ im öffentlich-rechtlichen „Konzern“, in: VergR, Heft 2, S. 185-193.

Gröpl, Christopher (2007), § 121 - Wirtschaftlichkeit und Sparsamkeit staatlichen Handelns, in: Handbuch des Staatsrechts: Band V: Rechtsquellen, Organisation, Finanzen, Band V, 3. Aufl., hrsg. von Josef Isensee und Paul Kirchhoff, Heidelberg, S. 1227-1263.

Hardraht, Karsten (2006), In-house-Geschäfte und europäisches Vergaberecht, Berlin.

Kirchner, Christian (2010), Zur Ökonomik des Vergaberechts, in: VergR, Heft 5, S. 725-735.

Leinemann, Ralf (2008), Umgang mit Spekulationspreisen, Dumpingangeboten und Mischkalkulationen, in: VergR, Heft 2 a, S. 346-352.

Mestwerdt, Thomas (2011), VOB/A 2009: Kommentar für die Bau- und Vergabepraxis, 2. Aufl., Berlin.

Walter, Sven Markus; Böhmann, Tilo; Krcmar, Helmut (2007): Industrialisierung der IT: Grundlagen, Merkmale und Ausprägungen eines Trends, in: HMD - Praxis der Wirtschaftsinformatik, Heft 256, S. 6-16.

Wildmann, Lothar (2010), Einführung in die Volkswirtschaftslehre, Mikroökonomie und Wettbewerbspolitik: Module der Volkswirtschaftslehre Band I: Module der Volkswirtschaftslehre 1, München. 